\title{
A quantitative analysis of illusion magnitude predicted by several averaging theories of the Müller-Lyer illusion
}

\author{
PATRICIA R. DELUCIA \\ Texas Tech University, Lubbock, Texas
}

\begin{abstract}
This article describes a quantitative method to evaluate several averaging (i.e., confusion and assimilation) theories by comparing predictions of the absolute magnitude of the Müller-Lyer (ML) illusion with results of previous studies of the composite ML figure. The magnitude of illusion was best predicted by Davies and Spencer's (1977) theory and by integrative field theory (Pressey \& Pressey, 1992). Furthermore, when the ML figure was at the point of subject equality, the average of shaft and intertip distances, and the configural dimensions proposed by Davies and Spencer, were most frequently closest to being equal in the apex-in ML and apex-out ML. Results indicate that a comparison of predicted and reported absolute magnitudes of the ML illusion can provide quantitative criteria to distinguish and evaluate averaging theories of the ML illusion.
\end{abstract}

The Müller-Lyer (ML) illusion (Müller-Lyer, 1889/ 1981) occurs with freely moving observers in normal spatial environments (see DeLucia \& Hochberg, 1991). It also occurs in spatial and temporal visual domains (Day \& Duffy, 1988; DeLucia \& Hochberg, 1985; Hochberg, 1968) and in the haptic mode (e.g., Over, 1966). The ML illusion is therefore relevant to theories of normal perception and to practical concerns about perceptual accuracy. Previous studies have ruled out perspective and orthogonalization theories, as well as low-level pattern explanations, of the ML illusion (e.g., DeLucia \& Hochberg, 1985, 1991).

The averaging theories, based on either confusion or assimilation among features in different locations of the figure, are still plausible, however. Such theories propose that observers respond not to the feature they are asked to judge but to other dimensions of the figure instead (for a summary, see Coren \& Girgus, 1978). Such dimensions include the distance between the centroids of areas enclosed by the fins, the average of or difference between shaft and intertip distances, and the assimilation of focal and contextual magnitudes; these dimensions are described in detail later. The occurrence of, and direction of change in the magnitude of, the ML illusion can be predicted successfully by any of these configural dimensions under at least a subset of stimulus conditions. As will be shown later, the configural dimensions on which these averaging theories are based are not independent. Thus, it would

I thank Kevin Jordan, Christopher W. Tyler, and an anonymous reviewer for comments on an earlier version. I also thank Alexander W. Pressey for providing a computer program to generate predictions of integrative field theory, and for enlightening discussions of his theory. Correspondence concerning this article should be addressed to $P$. R . DeLucia, Texas Tech University, Department of Psychology, Lubbock, Texas 79409-2051 (e-mail: djxpd@ttacs1.ttu.edu). seem that a comparison of predicted and reported direction of change in the magnitude of the ML illusion is not an adequate way to distinguish and assess predictions of the averaging theories. In this article, several theories will be evaluated through the comparison of predicted and reported absolute magnitudes of the illusion.

It may not yet be possible for the averaging theories to predict accurately the absolute magnitude of illusion. For example, it has been observed with respect to the quantitative integrative field theory (e.g., Pressey \& Pressey, 1992) that the patterns of data have been predicted remarkably well, but that predicting the absolute size of distortion seems hopeless at this time (Pressey, personal communication). However, testing predictions of absolute magnitude of illusion is more stringent than testing predictions of directional effects of various factors on the magnitude, and ultimately it may be more critical in the development of a successful theory (see Meehl, 1967). Thus, the objective of this article is to describe a method of determining which theories can predict the magnitude of illusion most closely, and thereby to provide quantitative criteria for comparing the theories. Averaging theories are particularly well suited to such a method, because they are based on quantifiable features of the ML figure.

\section{QUANTITATIVE ANALYSIS}

\footnotetext{
Method

A conceptual underpinning of the averaging theories in predicting the occurrence of the illusion is the comparison of a configural dimension (or dimensions) between the apex-in and apex-out ML figures (see Coren \& Girgus, 1978, p. 147). For example, a confusion theory based on the horizontal distance between the ends of the fins (intertip distance) predicts the ML illusion because the distance is larger in the apex-in ML than in the apex-out ML. This
} 
conceptual underpinning is taken further here, expressed as the following assumption, in generating predictions of illusion magnitude: if a configural dimension (or combination of dimensions) of the ML figure is the primary predictor of the illusion, the apparent difference between the two halves of the ML figure should approximate the actual difference in the dimension between the two halves of the ML figure.

Such an assumption, which is implicit in earlier work (Davies \& Spencer, 1977; Virsu, 1971), leads to two predictions that are tested in this article. (1) The percent difference in a proposed configural dimension, derived from the point of objective equality (POE), should approximate the percent magnitude of illusion. In Test 1 , for each configural dimension described below, the difference in the size of a dimension between apex-in and apex-out figures-derived from POE, was expressed as a percent of POE, for a sample of previous studies. The result was compared with the reported percent magnitude of illusion. (2) The size of a proposed configural dimension, derived from the point of subjective equality (PSE), should be approximately equal in the apex-in and apex-out figures. In Test 2 , for the same sample of studies, the difference in the size of a dimension between the apex-in and apexout figures-derived from the PSE-was expressed as a percent of POE and compared with zero. This is similar to a method used by Brunswik (1956), who reported that, at PSE, the "fringing area" of the variable figure was closer to the shaft length of the standard ML figure than was the shaft length of the variable ML figure.

The present quantitative method can be considered a hypotheticodeductive approach to describe formal relationships between objective properties of an illusory figure and the magnitude of illusion, aimed at ultimately explaining the illusion (see also Piaget, 1969). Although quantitative formulas alone do not provide an explanation of data, it is argued here that quantification of averaging hypotheses (see also Restle \& Decker, 1977), prediction of the absolute magnitude of the ML illusion (see also Meehl, 1967), and, ultimately, the specification of the stimulus conditions under which each theory can best predict the magnitude of illusion are important steps toward predicting and explaining the illusion.

This article focuses on the development of a quantitative method to evaluate several averaging theories by comparing predictions of absolute magnitude of the ML illusion with the reported magnitude of illusion. The method is applied to a sample of previous studies of a conventional ML figure. If the method is deemed useful, application to a much wider range of ML patterns would be a prudent next step toward providing a more complete analysis of the averaging theories. The studies were chosen because they represent a variety of stimulus conditions (e.g., conventional line drawings of different sizes, small and large solid objects, stationary and moving observers, full and aperture viewing), and because they utilized the composite ML figure. The latter, which was reported as the most popular variant (Coren \& Girgus, 1978, p. 30), facilitated estimates of the attentive field needed to analyze integrative field theory (Pressey \& Pressey, 1992).

Proposed configural dimensions. Configural dimensions proposed as the bases of five theories of the ML illusion are described below and in Figure 1. Refer to Figure 1A for notation.

Centroid theories are based on the assumption that observers respond to the distance between the centroids of triangular areas formed by connecting the ends of each pair of fins with a vertical line (see Restle \& Decker, 1977; and Virsu, 1971). As represented in Figure 1B, the centroid distance in the apex-in $\mathrm{ML}$ is greater than that in the apex-out ML, thus predicting the illusion.

Erlebacher and Sekuler (1969) reported that when the horizontal distance between the ends of the fins (intertip distance) remained constant and fin angle varied, the magnitude of the apex-out ML illusion remained constant. After a series of experiments, the authors concluded that the perception of line length in the apex-out ML figure is a compromise between actual length and intertip distance (see also Sekuler \& Erlebacher, 1971). This compromise, quantified here

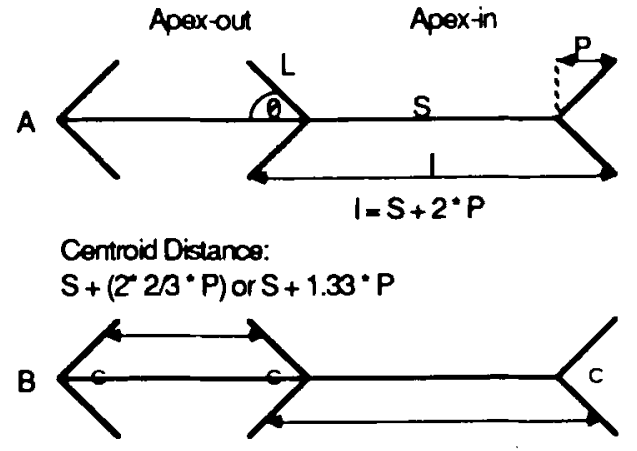

Average of Shat \& Intertip Distances: $(S+1) / 2 \propto S+P$
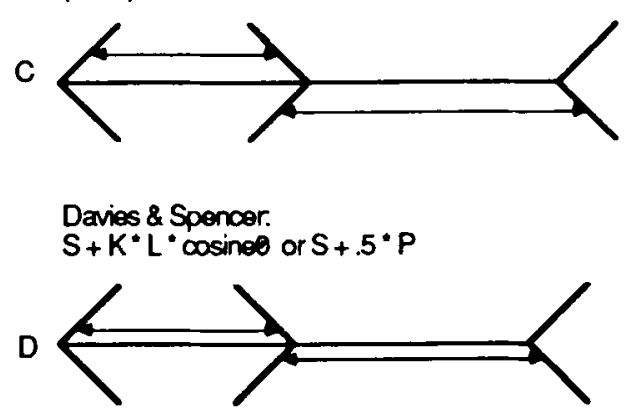

Figure 1. Schematic representation of averaging theories: (A) Notation. (B) Centroid distance. (C) Average of shaft and intertip distances. (D) Davies and Spencer (1977). Formulas represent prediction of the point of subjective equality for the apex-out figure; reverse the sign for the apex-in figure.

as the average of the shaft and intertip distances and represented in Figure 1C, is applied to the apex-in and apex-out ML (see also McClellan, Bernstein, \& Garbin, 1984). However, when intertip distance (and shaft length) is held constant, the centroid distance and the configural dimensions proposed by Piaget (1969) and Davies and Spencer (1977) also remain constant (to be discussed later). These dimensions are tested independently here. (Note-Predictions of illusion magnitude based on intertip distance alone or $2 \mathrm{P}$ - see also McClellan et al., 1984-were not as successful as the other predictions considered here, and thus are not discussed.)

Davies and Spencer (1977) suggested that the error in the ML illusion can be predicted by the formula, $K * L * \operatorname{cosine} \theta$, where $K, L$, and $\theta$ denote a constant value, fin length, and fin angle, respectively. The term $L *$ cosine $\theta$ is equivalent to $(I-S) / 2$ or $P$, and Davies and Spencer estimated $K$ to be .5 for composite ML figures such as those analyzed here. Thus, $K * L * \operatorname{cosine} \theta$ is equivalent to one fourth the difference between the shaft and intertip distances. If this formula represents the error in the ML illusion, it is reasonable to propose that observers "confuse" the configural dimension shown in Figure 1D with the shaft. Thus, Davies and Spencer's model is applied here as a confusion theory, without assuming their explanatory mechanism of a perceptual distortion at the apices of the fins.

Davies and Spencer's (1977) formula predicts no ML illusion in figures with rectangular fins or $90^{\circ}$ arcs; in such figures, the illusion occurs but is smaller in magnitude (Coren \& Girgus, 1978). It would be premature to discard Davies and Spencer's formulations, however, because currently no single stimulus factor can ac count for the ML illusion under all stimulus configurations (see McClellan et al., 1984), and it would be useful to identify the con- 
ditions under which a theory can successfully predict the magnitude of illusion.

The assimilation of focal and contextual magnitudes, along with the location of the contextual magnitudes within attentive and interactive fields, is the basis of integrative field theory (Pressey \& Pressey, 1992). In the present analysis, predictions of integrative field theory were generated with a model that included only an attentive field, which resulted in better predictions than did a model with both attentive and interactive fields. This may have occurred because the stimuli analyzed here do not match the prototypical configuration used by integrative field theory (albeit the attentive and interactive fields were estimated by maintaining constant ratios of the appropriate stimulus parameters used in Pressey's earlier studies). It is not clear how to specify the interactive field in such cases more accurately (Pressey, personal communication).

Whereas the above models are based on confusion or assimilation hypotheses, Piaget (1969) proposed that many geometrical illusions are due to contrast or to an accentuation of inequalities in a figure - that is, to the relationship between a small part of a figure and the difference between it and a larger part of the figure. For example, he asserted that the short and long sides of a trapezoid are typically overestimated and underestimated, respectively, because of a misperception of the undrawn length that represents the difference between the long and short sides of the trapezoid. Piaget considered the ML illusion to be a "double trapezium effect," with the relationship between $2 P$ and $I$ a critical factor. In the present analysis, the ratio $(I-S) / I$, which is equivalent to (2 * $P) / I$, is used to represent the general principle proposed by $\mathrm{Pi}$ aget (see Piaget, 1969, p. 67, Table 16B). Piaget's quantified contrast model is included here because it allows prediction of the $a b$ solute magnitude of illusion. The predictive ability of Piaget's model is assessed without any further attempt to compare assimilation and contrast explanations of the illusions, a distinction that Piaget tried to go beyond in developing general explanations in terms of inequalities in the dimensions of a figure (p. 39).

Covariation of the configural dimensions. The configural dimensions on which the above theories are based are not independent. Specifically, for all theories, the predicted magnitude of constant error in the ML illusion can be expressed as a proportion of $P$ (see Figure 1). For the average of shaft and intertip distances, the proposed error is $P$; for the centroid hypothesis, it is $1.33 *$ $P$; for Davies and Spencer's (1977) hypothesis, it is $.5 * P$, and for Piaget's (1969) hypothesis, it is $2 * P / I$ [or $P /(.5 * I)]$. In the integrative field theory, the illusion is predicted by the difference between the standard line and a contextual magnitude (which can be expressed as a proportion of $P$ ), multiplied by the probability that the contextual magnitude contributes to the judgment of the standard line. The probability is estimated by the contextual magnitude's position within the attentive and interactive fields. In the ML illusion, a number of contextual magnitudes are sampled, and a weighted averaged is computed.

The mean percent illusion magnitude reported in the following studies was included in the previously described analyses: Carrasco (1986, Experiment 1); Davies and Spencer (1977; fin length was $15 \%$ of shaft); Day and Duffy (1988, Experiment 1; a moving ML figure viewed through a slit); DeLucia and Hochberg (1991; solid cones, large wood construction, drawn figure and book constructions in which the ML figure was oriented to have nonconvergent fins); Dewar (1967; fin lengths of 1, $4 \mathrm{~cm}$ ); Heymans (1896; see Berliner, 1948); Judd (1905, Day 1); Mack, Heuer, Villardi, and Chambers (1985, Experiment 1).

\section{Results}

Test 1. At POE, does the percent difference in the configural dimension between the two halves of the ML figure approximate the percent illusion magnitude?
The results of Test 1 are shown in Figure 2. Integrative field theory and Piaget's (1969) theory typically underestimated the magnitude of illusion, whereas the other theories typically overestimated it. The correlation between the predicted and reported magnitudes of the ML illusion was significantly different from zero $(p<.02$, two-tailed) for all theories except Piaget's. Pearson $r$ values were .52 for all except integrative field theory, for which $r=.4$. A significant correlation between the predicted and reported magnitudes of the illusion seems a minimal requirement for any theory, and thus Piaget's formulations will not be considered further. The comparable correlations among the averaging theories are not surprising, because all of the theories can predict direction of change in the magnitude of illusion, and all predictions are based on a proportion of $P$.

Additional analyses indicate theories that predicted the absolute illusion magnitude most closely. Bonferroni $t$ tests for the difference between sample and predicted means indicated that, for all theories, such differences were typically greater than zero $(p<.05)$. Considering all of the sampled studies (except Carrasco, 1986; Dewar, 1967; and Davies \& Spencer, 1977, in which raw scores or standard deviations were not reported), the frequency with which any theory's predictions "matched" the reported illusion was never more than chance probability. Furthermore, for each study, the theories were ranked by the amount of deviation between predicted and reported magnitude of illusion. The frequency with which each theory's prediction resulted in the smallest deviation was computed. The frequencies were 16, 17, 1, 0 (and 1 tie) for the integrative field, Davies and Spencer's (1977), the average of shaft and intertip, and the centroid theories, respectively. Chi-square goodness-of-fit tests indicated significant differences among such frequencies $\left[\chi^{2}(3)=\right.$ $29.38, p<.001]$, but not between Davies and Spencer's frequencies and integrative field theory's frequencies. Finally, the root mean square error (RMSE) was used as another measure of the relative accuracy with which theories predicted the absolute magnitude of illusion. The RMSE values were 8.71, 9.41, 27.52, and 40.82 for the four theories, respectively. These results indicate that integrative field theory and Davies and Spencer's theory most frequently predicted a magnitude of illusion that was closest to the reported magnitude.

Test 2. At PSE, is the configural dimension approximately equal in both halves of the ML figure?

The percent difference in each configural dimension between the apex-in and apex-out figures, derived from the PSE, is shown in Figure 3 and was compared with zero. The mean deviation from zero was $12.83 \%, 12.15 \%$, $18.45 \%$, and $21.22 \%$ for Davies and Spencer's (1977), the average of shaft and intertip, the integrative field, and the centroid theories, respectively. All were significantly greater than zero, $p<.001$ ( $t$ values ranged from 4.9 to 8.4, with $d f$ of 34 ). The RMSE values were 16.47 , $18.81,22.51$, and 29.39 for the four theories, respectively. Furthermore, the frequencies with which a dimen- 


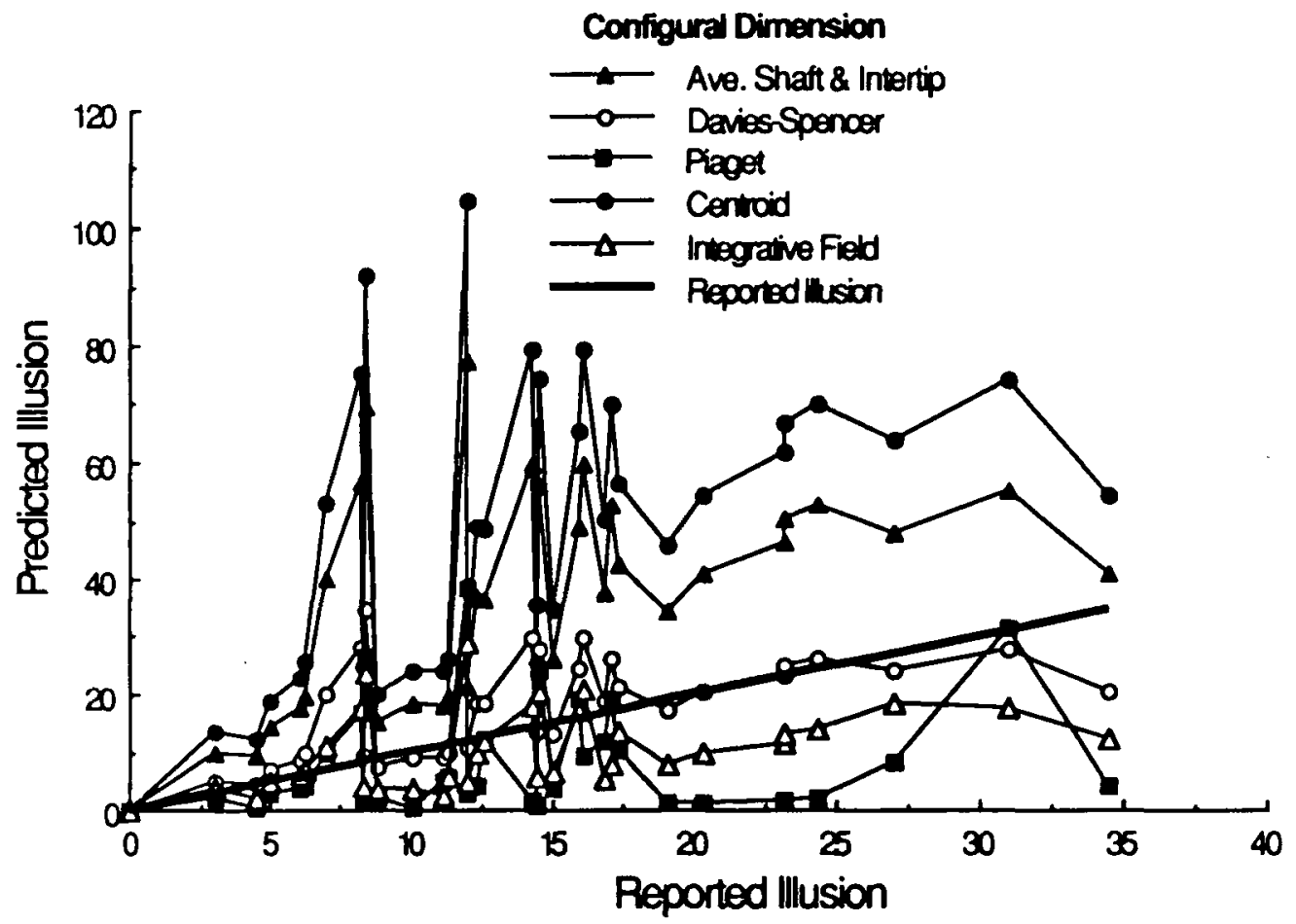

Figure 2. The difference in the magnitude of each configural dimension between apex-in and apex-out MüllerLyer (ML) figures derived from the point of objective equality (expressed as a percentage of POE), compared with the reported percent magnitude of illusion in the following studies: DeLucia and Hochberg (1991; solid cones, large wood construction, drawn figure and book constructions in which the ML figure was oriented to have nonconvergent fins); Mack, Heuer, Villardi, and Chambers (1985, Experiment 1); Davies and Spencer (1977; fin length was 15\% of shaft); Heymans (1896; see Berliner,'1948); Day and Duffy (1988; Experiment 1; a moving ML figure viewed through a slit); Judd (1905, Day 1); Carrasco (1986, Experiment 1); Dewar (1967; fin lengths of $1,4 \mathrm{~cm}$ ).

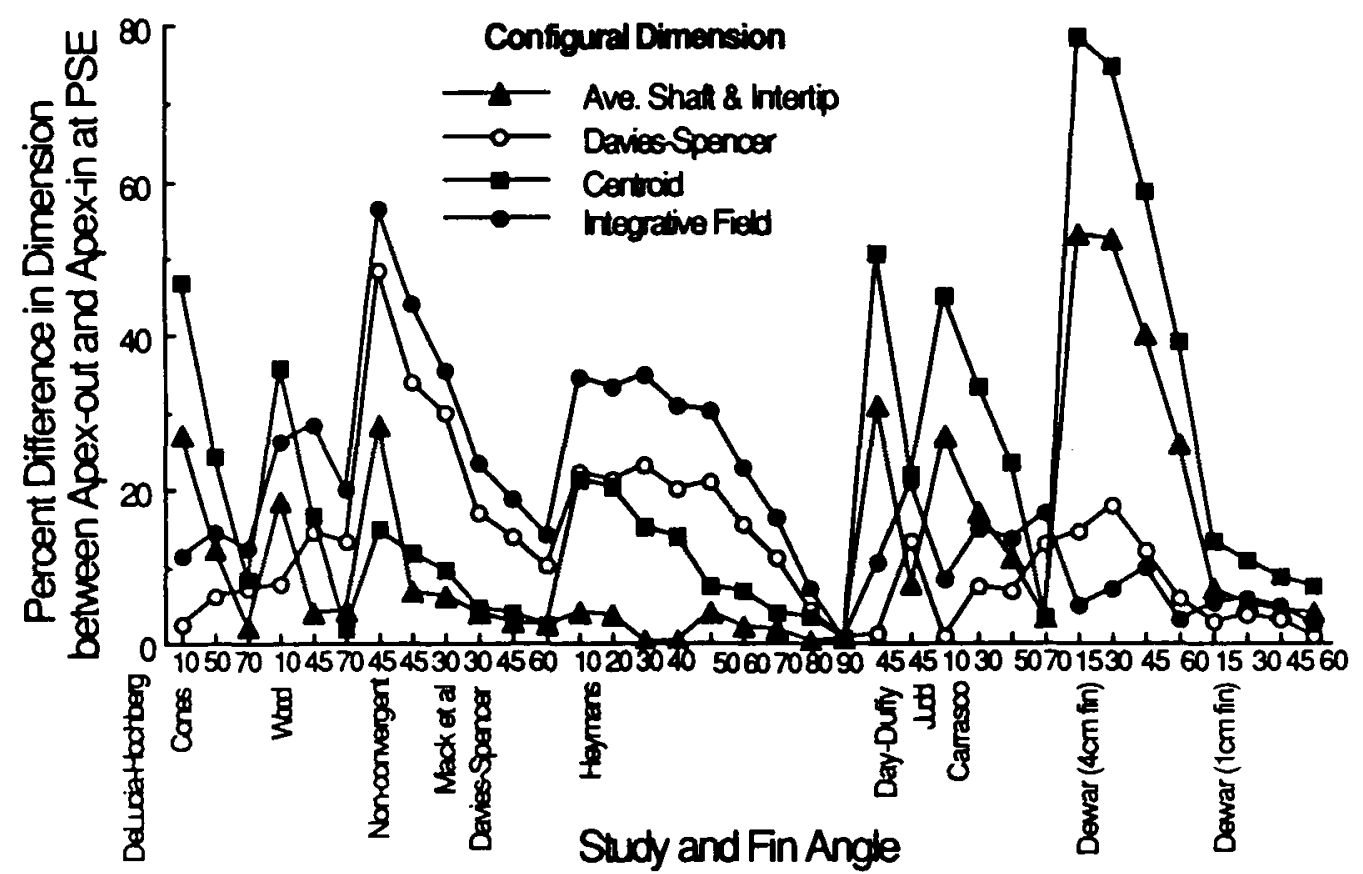

Figure 3. The difference in the size of each dimension between apex-in and apex-out Müler-Lyer figures derived from the point of subjective equality (expressed as a percentage of the point of objective equality) for the selected studies. 
sion was closest to being equal in the apex-in and apexout ML figures were $11,15,4$, and 3 (and 2 ties) for Davies and Spencer's, the average of shaft and intertip, the integrative field, and the centroid theories, respectively. Chi-square goodness-of-fit tests indicated significant differences among such frequencies $\left[\chi^{2}(3)=11.48, p<\right.$ .02 J, but not between the frequencies for the Davies and Spencer and average of shaft and intertip hypotheses. These results indicate that the dimensions proposed by the latter theories were most frequently closest to being equal in the apex-in ML and apex-out ML when the dimensions were derived from the PSE.

\section{DISCUSSION}

All theories considered here predicted a magnitude of illusion that differed significantly from the reported magnitude. However, the dimensions proposed by integrative field theory, and Davies and Spencer's (1977) theory, most frequently predicted illusion magnitudes that were closest to the reported magnitude. Furthermore, each of the proposed configural dimensions was significantly different from being equal in both halves of the ML figure when derived from the PSE. However, the average of shaft and intertip distances and the configural dimensions proposed by Davies and Spencer were most frequently closest to being equal in the apex-in ML and apex-out ML.

One could argue that because the model proposed by Davies and Spencer (1977) is the only one that fared relatively successfully in both Test 1 and Test 2 , their model best fits the data; that is, $.5 P$ best predicts the magnitude of error. Additional analyses of a wider variety of stimuli is needed before such a conclusion can be reached, however. At the least, the results indicate that predictions of the magnitude of illusion (from the POE) best fit the data when the constant error is assumed to be of a magnitude between that proposed by Davies and Spencer's model (which overestimates) and that proposed by integrative field theory (which underestimates). Furthermore, the results suggest that quantitative analyses that focus on predictions of absolute magnitude of the ML illusion can be useful in distinguishing and evaluating predictions of the averaging theories.

However, one could argue that the generality of this analysis is limited to composite ML figures: The analysis is based on the assumption that the percent magnitude of illusion should be approximately equal to the percent difference between a proposed configural dimension in the two halves of the ML figure. The analysis also assumes implicitly, therefore, that the configural dimension affects the judgment of each half of the ML figure in the same way. Such assumptions may be questioned in light of the asymmetry reported in the ML illusion; that is, the two halves of the ML may be different illusions (Bross, Blair, \& Longtin, 1978; Butchard \& Pressey, 1971; Christie, 1975; Cooper \& Runyon, 1970; Day \& Dickinson, 1976; Erlebacher \& Sekuler, 1974; GreistBousquet \& Schiffman, 1981; Piaget, 1969; Sekuler \&
Erlebacher, 1971). Proponents of such a perspective assert that stimuli which combine both ML figures-such as those analyzed in this article-should be avoided (Sekuler \& Erlebacher, 1971; see also Lewis, 1909).

To abandon the present quantitative approach because of asymmetry data would be premature for several reasons: First, the evidence that the ML consists of two fundamentally different illusions is equivocal. It has been reported that asymmetries in the data may be partly due to a methodological artifact of the standard or control line (Beagley, 1985; Christie, 1975; see also Adam \& Bateman, 1980), to the different spatial locations of the contextual stimuli in the two ML forms (Pressey \& Smith, 1986), or to differences in attention between the two ML forms (Pressey \& Pressey, 1992). Indeed it has been suggested that a single mechanism underlies the apex-in and apex-out ML illusion (Brigell \& Uhlarik, 1979; Jordan \& Uhlarik, 1986; Worrall \& Firth, 1974). Second, in asymmetry studies, subjects have necessarily compared the length of one half of the ML with a line (or with the distance between two points); each half of the ML was judged separately. It is difficult to see how asymmetry can be measured in a combined ML figure. If the ML consists of two independent illusions as based on asymmetry studies, the present analysis may not apply to such versions of the ML figure. The analysis would not necessarily be invalid, however, for composite ML figures. Finally, the pattern of predictions generated by the present analysis reflects the pattern of results from the selected studies. Nevertheless, an exploratory experiment was completed to determine whether the illusion obtained when subjects compare each half of the ML figure with a line is different from the illusion obtained when subjects compare the two halves of the ML figure with each other. If so, the results of the present quantitative analysis may be limited to composite ML figures.

\section{EXPERIMENT}

If combined forms of the ML figure cause the presumably different apex-in and apex-out ML illusions to interact and thus cause a confounded measure of the illusion, it is reasonable to predict the following: The magnitude of the illusion obtained when subjects compare each half of the ML figure with a line (ML-line judgment) should be different from the magnitude of illusion obtained when subjects compare the apex-in ML with the apex-out ML (ML-ML judgment). This prediction was tested in the following experiment.

\section{Method}

Subjects. Eight Texas Tech University students who had normal or corrected visual acuity participated as part of a psychology course requirement; they were naive as to the experimental hypotheses.

Stimuli. A slide-rule construction was used to create eight ML figures, drawn with black ink on $21.6 \times 27.9 \mathrm{~cm}$ white paper, and represented in Figure $4:(1 \& 2)$ a fixed apex-out ML with a variable plain line, and vice versa; ( $3 \& 4)$ a fixed apex-in ML with 


\section{ML-Line Judgments}

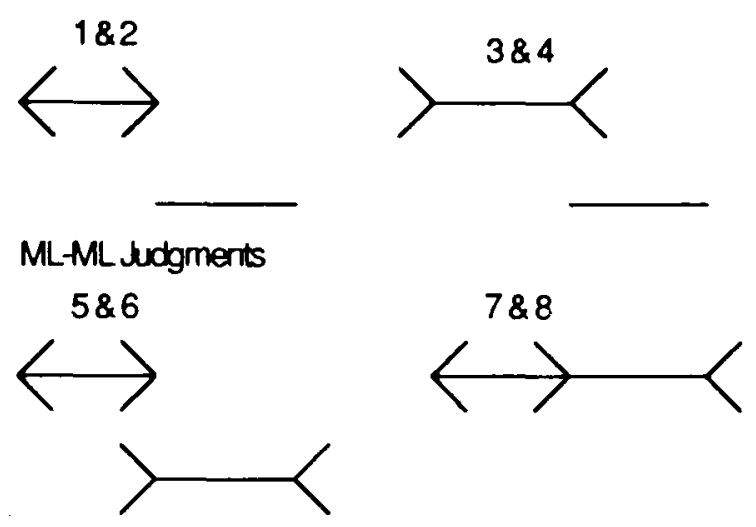

Figure 4. Schematic representation of the stimuli used in this study.

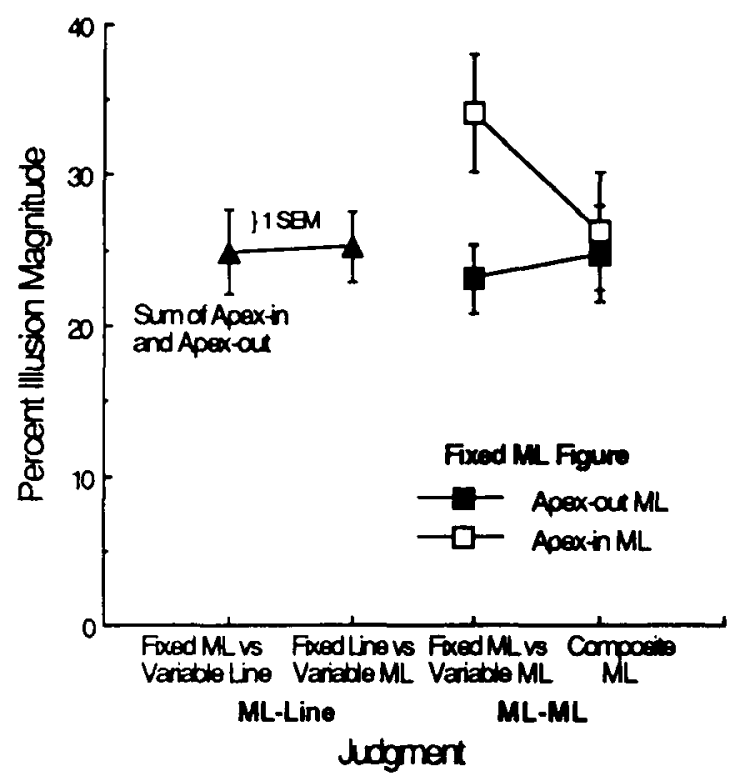

Figure 5. Mean percent illusion magnitude for each figure (the mean illusion in Figures 1 and 2 and in Figures 3 and 4 were summed). SEM, 1 standard error of mean.

a variable plain line, and vice versa; $(5 \& 6)$ a fixed apex-out $M L$ with a variable apex-in $M L$, and vice versa; $(7 \& 8)$ a composite ML figure with a fixed apex-in ML, and with a fixed apex-out ML. There were four pairs of figures, and the members of each pair differed only by reversing which portion of the figure was adjusted by the subjects. Fins were always $2.8 \mathrm{~cm}$ long and formed a $30^{\circ}$ angle to the shaft; the POE was always $10.1 \mathrm{~cm}$.

Design and Procedure. The PSE was measured with an adjustment method in which the initial position of the variable length was set to either a maximum or a minimum on half the trials. Each subject viewed all figures four times in a unique random order, with orientation of figure (for half of the subjects, the figures were turned upside down) counterbalanced across subjects.

\section{Results and Discussion}

The results are summarized in Figure 5. Magnitude of illusion was defined as the constant error expressed as a percentage of the POE. To determine whether the ML-line judgments resulted in a different illusion from the ML-ML judgments, the means of percent illusion magnitude that each subject obtained in Figures 1 and 3 were summed (similarly for Figures 2 and 4); the sums were used in the analysis below, resulting in six conditions. The sums reflect the error due to both halves of the ML figure, and thus allow an appropriate comparison with the illusion measured in the combined figures.

A $6 \times 2$ (figure $\times$ orientation) analysis of variance indicated a significant effect only of figure $[F(5,30)=$ $3.03, p<.03]$. Tukey's HSD tests indicated that the illusion obtained when subjects adjusted the apex-out ML to match the fixed apex-in ML was significantly greater $(p<.05)$ than the illusion obtained when the apex-in ML was adjusted to match the fixed apex-out ML. Such an adjustment asymmetry in this pair of figures alone cannot be explained without further study. More importantly, the total magnitude of illusion from the ML-line judgments did not differ significantly from the illusion obtained with any of the combined ML figures. Notwithstanding the caveat that this is a null result, the data suggest that-at least for the figures used here, the total magnitude of illusion obtained when each half of the ML figure is compared with a line does not differ significantly from the illusion obtained when the two halves are viewed in a combined figure. These results suggest that the present quantitative analysis is not limited to combined ML figures as could be suggested by results of asymmetry studies.

\section{CONCLUSIONS}

The results indicate that a quantitative method that compares data with predictions of the absolute magnitude of the ML illusion generated from configural dimensions on which averaging theories are based can be used to evaluate such theories. Models that predict a magnitude of constant error between that proposed by Davies and Spencer's (1977) model and that proposed by integrative field theory deserve further exploration toward the aim of predicting the magnitude of the ML illusion. Future applications of this quantitative method should include a greater number and variety of stimulus conditions and may lead to combinations of dimensions (see McClellan et al., 1984) that better predict the absolute magnitude of the illusion, as well as to the specification of the stimulus conditions under which each averaging theory can best predict the magnitude of the illusion.

\section{REFERENCES}

AdAm, J., \& Bateman, L. (1980). Control stimuli in investigations of the acute-angled and obtuse-angled Müller-Lyer illusions. Perception, 9, 467-474.

BeAGLEy, W. K. (1985). Interaction of Müller-Lyer with filled-unfilled space illusion: An explanation of Müller-Lyer asymmetry. Perception \& Psychophysics, 37, 45-49.

Berliner, A. (1948). Lectures on visual psychology. Chicago: Professional Press.

Brigell, M., \& Uhlarik, J. (1979). The relational determination of length illusions and length aftereffects. Perception, 8, 187-197. 
Bross, M., Blair, R., \& Longtin, P. (1978). Assimilation theory, attentive fields, and the Müller-Lyer illusion. Perception, 7, 297-304.

Brunswik, E. (1956). Perception and the representative design of psychological experiments. Los Angeles: University of California Press.

Butchard, N., \& Pressey, A. W. (1971). The effects of closing lines on the two forms of the Müller-Lyer illusion. Psychonomic Science, 23, 257-258.

Carrasco, M. (1986). A test of the spatial frequency explanation of the Müller-Lyer illusion. Perception, 15, 553-562.

Christie, P. S. (1975). Asymmetry in the Müller-Lyer illusion: Artifact or genuine effect? Perception, 4, 453-457.

COOPER, M. R., \& RUNYON, R. P. (1970). Error increase and decrease in minimal form of Mueller-Lyer illusion. Perceptual \& Motor Skills, 31, 535-538

COREN, S., \& Girgus, J. S. (1978). Seeing is deceiving: The psychology of visual illusions. New Jersey: Erlbaum.

DAviES, T., \& SPENCER, J. (1977). An explanation for the Mueller-Lyer illusion. Perceptual \& Motor Skills, 45, 219-224.

DAY, R. H., \& Dickinson, R. G. (1976). Apparent length of the arms of acute and obtuse angles, and the components of the Müller-Lyer illusion. Australian Journal of Psychology, 28, 137-148.

DAY, R. H., \& DUfFY, F. M. (1988). Illusions of time and extent when the Müller-Lyer figure moves in an aperture. Perception \& Psychophysics, 44, 205-210.

Delucia, P., \& HochberG, J. (1985). Illusions in the real world and in the mind's eye. Proceedings and Abstracts of the Annual Meeting of the Eastern Psychological Association, 56, 38.

DeLuCia, P. R., \& HochrerG, J. (1991). Geometrical illusions in solid objects under ordinary viewing conditions. Perception \& Psychophysics, 50, 547-554.

DEwar, R. E. (1967). Stimulus determinants of the magnitude of the Mueller-Lyer illusion. Perceptual \& Motor Skills, 24, 708-710.

Erlebacher, A., \& Sekuler, R. (1969). Explanation of the MuellerLyer illusion: Confusion theory examined. Joumal of Experimental Psychology, 80, 462-467.

Erlebacher, A., \& Sexuler, R. (1974). Perceived length depends on exposure duration: Straight lines and Müller-Lyer stimuli. Journal of Experimental Psychology, 103, 724-728.

Greist-Bousquet, S., Schifrman, H. R. (1981). The many illusions of the Müller-Lyer: Comparisons of the wings-in and wings-out illusions and manipulations of standard and dot forms. Perception, 10, 147-154.

Hochrerg, J. (1968). In the mind's eye. In R. N. Haber (Ed.), Contemporary theory and research in visual perception (pp. 309-331). New York: Holt, Rinehart \& Winston.
JoRDAN, K., \& UhlaRik, J. (1986). Length contrast in the Müller-Lyer figure: Functional equivalence of temporal and spatial separation. Perception \& Psychophysics, 39, 267-274

Judd, C. H. (1905). The Mueller-Lyer Illusion. Psychological Review Monograph Supplements, 7, 55-81.

LEWIS, E. O. (1909). Confluxion and contrast effects in the MuellerLyer illusion. British Journal of Psychology, 3, 21-41.

Mack, A., Heuer, F., Villardi, K., \& Chambers, D. (1985). The disassociation of position and extent in Müller-Lyer figures. Perception \& Psychophysics, 37, 335-344.

McClellan, P. G., Bernstein, I. H., \& Garbin, C. P. (1984). What makes the Mueller a liar: A multiple-cue approach. Perception \& Psychophysics, 36, 234-244.

MeEHL, P. E. (1967). Theory-testing in psychology and physics: A methodological paradox. Philosophy of Science, 34, 103-115.

MüLLER-LyER, F. C. (1889). Zur Lehre von den optischen Täuschungen: Über Kontrast und Konfluxion. Zeitschrift für Psychologie, 9. 1-16. [Also translated in R. Day \& H. Knuth (Eds.) (1981), The contributions of F. C. Müller-Lyer. Perception, 10, 126-146.]

OVER, R. (1966). A comparison of haptic and visual judgements of some illusions. American Journal of Psychology, 79, 590-595.

Piaget, J. (1969). The mechanisms of perception. New York: Basic Books.

Pressey, A. W., \& Pressey, C. A. (1992). Attentive fields are related to focal and contextual features: A study of Müller-Lyer distortions. Perception \& Psychophysics, 51, 423-436.

Pressey, A. W., \& Smith, N. E. (1986). The effects of location, orientation, and cumulation of boxes in the Baldwin illusion. Perception \& Psychophysics, 40, 344-350.

Restle, F., \& Decker, J. (1977). Size of the Müller-Lyer illusion as a function of its dimensions: Theory and data. Perception \& Psychophysics, 21, 489-503.

Sekuler, R., \& Erlebacher, A. (1971). The two illusions of MuellerLyer: Confusion theory reexamined. American Journal of Psychology, 84, 477-486.

VIRSU, V. (1971). Tendencies to eye movement, and misperception of curvature, direction, and length. Perception \& Psychophysics, 9, 65-72.

Worrall, N., \& FirTh, D. (1974). The components of the standard and reverse Müller-Lyer illusions. Quarterly Journal of Experimental Psychology, 26, 342-354.

(Manuscript received January 17, 1992; revision accepted for publication October $21,1992$. 